\title{
Dynamic Power Balance Analysis in JET
}

\author{
G.F.Matthews ${ }^{\mathrm{a}^{*}}$, S. A. Silburn ${ }^{\mathrm{a}}$, C. D. Challis ${ }^{\mathrm{a}}$, T.Eich ${ }^{\mathrm{b}}$, D. Iglesias ${ }^{\mathrm{a}}$, D. King ${ }^{\mathrm{a}}$, \\ B.Sieglin ${ }^{\mathrm{b}}$ and JET Contributors ${ }^{\mathrm{c}}$ \\ EUROfusion Consortium, JET, Culham Science Centre, Abingdon, OX14 3DB, UK \\ ${ }^{a}$ CCFE, Culham Science Centre, Abingdon, OX14 3DB, UK \\ ${ }^{b}$ Max-Planck-Institut fur Plasmaphysik, Boltzmannstrasse 2, D-85748 Garching, Germany \\ 'See the author list of "Overview of the JET results in support to ITER" by X. Litaudon et al. to be \\ published in Nuclear Fusion Special issue: overview and summary reports from the 26th Fusion Energy \\ Conference (Kyoto, Japan, 17-22 October 2016) \\ *Corresponding author: tel.: 00441235 464523,gfm@ukaea.uk (G.F.Matthews)
}

\begin{abstract}
The full scale realization of nuclear fusion as an energy source requires a detailed understanding of power and energy balance in current experimental devices. In this we explore whether a global power balance model in which some of the calibration factors applied to the source or sink terms are fitted to the data can provide insight into possible causes of any discrepancies in power and energy balance seen in the JET tokamak. We show that the dynamics in the power balance can only be properly reproduced by including the changes in the thermal stored energy which therefore provides an additional opportunity to cross calibrate other terms in the power balance equation. Although the results are inconclusive with respect to the original goal of identifying the source of the discrepancies in the energy balance, we do find that with optimized parameters an extremely good prediction of the total power measured at the outer divertor target can be obtained over a wide range of pulses with time resolution up to $\sim 25 \mathrm{~ms}$.
\end{abstract}

Keywords: Fusion energy, JET, power balance, energy balance, IR thermograph

PACS: 52.55.Fa, 52.55.Rk

\section{Introduction}

In order to design a tokamak reactor capable of fusion energy production on the scale required for electricity generation, we need the ability to predict the power and energy balance in detail to ensure that all the components have sufficient thermal capability [12]. This paper aims to check the empirical self-consistency of the JET power balance for a wide parameter range of typical attached plasmas as an important baseline against which the power balance in highly radiating scenarios can be judged [13].

At the PSI conference in Rome (2016) an energy balance analysis based on tile thermocouples was presented [1] for JET's ITER-like Wall [2]. This revealed that typically, 25\% of the nominal energy input was unaccountable when compared to the sum of the losses. This fractional discrepancy was found to be similar in both low and high radiated power fraction plasmas [1]. To help track down the terms responsible for this discrepancy, a regression of the data was carried out to find combinations of correction factors (multipliers) most consistent with the energy balance using equation (1) below;

$$
\mathbf{a} \times E_{\mathrm{Oh}}+\mathbf{b} \times \mathrm{E}_{\mathrm{NBI}}+\mathbf{c} \times \mathrm{E}_{\mathrm{ICH}}=\mathbf{d} \times\left(\mathrm{E}_{\mathrm{TCdiv}}+\mathrm{E}_{\mathrm{TClim}}\right)+\mathbf{e} \times\left(\mathrm{E}_{\mathrm{R}}-\mathrm{f}_{\mathrm{B}} \mathrm{E}_{\mathrm{RB}}-\mathrm{f}_{\mathrm{X}} \mathrm{E}_{\mathrm{RX}}\right)
$$

Parameters $\mathbf{a}, \mathbf{b}, \mathbf{c}$ are multipliers for the energy sources (Ohmic input, neutral beam input and ion cyclotron heating) and $\mathbf{d}$, e are multipliers for the energy loss terms (thermocouple based energies for the limiters and divertor and main chamber radiation). The multipliers $f_{B}$ and $f_{X}$ on main chamber radiation $\left(\mathrm{E}_{\mathrm{RB}}\right)$ and $\mathrm{X}$-point radiation $\left(\mathrm{E}_{\mathrm{RX}}\right)$ are there to avoid double counting of radiation losses by the tile thermocouples. One or more of the multipliers a to e must be fixed when fitting the data. If a particular fitted multiplier is not as expected then this can be due to a calibration error or that an unmeasured energy source or sink which is co-linear with this particular term.

Unfortunately, the results of the energy balance analysis [1] were inconclusive with respect to identifying the cause of the energy deficit. However, a simple calibration error on the radiated power was shown to be the least likely explanation for the deficit. The more favoured fits suggested an error which was co-linear with the neutral beam injection heating (NBI). However, a detailed error analysis 
for the NBI system suggests an error of not more than $10 \%$ in the power calculation while $20 \%$ reduction would be required to match the average energy balance.

The origin of these discrepancies matters because it affects the interpretation of many experiments from L-H transition studies to the study of scenarios with high radiated power fraction as foreseen for DEMO. One challenge for energy balance analysis is to understand the errors in the calibrations of the energy source terms (plasma heating) and the sink terms such as total radiated energy and energy conducted to the plasma facing components via the plasma. Another challenge is to identify potential losses which are not measured directly such as those due to charge exchange.

In this new work, we extend the methodology applied to the energy balance to consider the power balance in JET. The disadvantage of doing this is that thermocouple based tile calorimetry is a simple method which while only providing one data point per pulse from the cooldown, is expected to be very reliable in terms of absolute calibration. This is in contrast to the JET infra-red system which can be complicated to interpret due to issues of uncertain surface emissivity, background light and a viewing area usually limited to the outer strike point. However, power balance can yield many more points per pulse covering a wider parameter range than energy balance where everything is shot integrated. As we will show, power balance analysis also benefits from the fact that fluctuations in thermal stored energy of the plasma due to confinement changes and ELMs adds a new term which can rival the neutral beam heating in its contribution to power balance on timescales up to the energy confinement time.

A similar study of energy balance in Tore Supra using its active cooling system for calorimetry [10] showed excellent energy accounting but only after the RF input powers were revised downwards in light of the calorimetry results. Power balance during ELMs was studied in ASDEX-Upgrade [9] but with the aim of understanding how much ELM load goes outside the divertor. Generally, quantitative assessment of the quality of power and energy balance in tokamaks has been avoided due to the difficulty in assessing the uncertainties in all the contributing terms.

\section{Dynamic power balance analysis}

To carry out a power balance analysis we match the sum of the power inputs on the left to the total losses on the right-hand side of equation (2). The analysis has been termed dynamic in that we are not selecting quasi-steady state time intervals and are specifically interested in maximising the variations of the different terms so we can reliably evaluate the multipliers.

$$
\mathbf{a} \times \mathrm{P}_{\mathrm{Oh}}+\mathbf{b} \times \mathrm{P}_{\mathrm{NBI}}+\mathbf{c} \times \mathrm{P}_{\mathrm{ICH}}=\mathbf{d} \times \mathrm{P}_{\mathrm{IR}}+\mathbf{e} \times \mathrm{P}_{\mathrm{R}}+\mathbf{f} \times \mathrm{dW} / \mathrm{dt}
$$

The Ohmic input power is $\mathrm{P}_{\mathrm{Oh}}$, the absorbed neutral beam power (allowing for shinethrough) is $\mathrm{P}_{\mathrm{NBI}}$ and the absorbed ion cyclotron heating power is $\mathrm{P}_{\mathrm{ICH}}$. Parameters $\mathbf{a}, \mathbf{b}, \mathbf{c}$ when fitted can allow for calibration errors in nominal heating powers or co-linear power losses which are not otherwise measured. The process can be thought of as cross calibration of unknowns against knowns.

On the loss side of equation (2) we start with the total power to the outer strike point measured by infra-red cameras $P_{\text {IR }}$ [3]. Multiplier $\mathbf{d}$ represents both the ratio of the total power conducted from the plasma to that observed by the IR camera and also any calibration errors or co-linear losses. The next loss term is the total radiated power $P_{R}$ as measured by bolometer systems and estimated using a fast inter-shot algorithm [4]. Because the IR analysis is restricted to a relatively small area of the divertor near the outer strike point, we no longer include the double counting term which appears in the energy balance equation (1). This difference arises because the divertor tile thermocouples allow us to measure the energy deposited over a large surface area where the contribution from plasma radiation can be significant and must therefore be discounted.

The final loss term in (2) relates to changes in plasma thermal stored energy (W). JET measures stored energy using diagnostics that measure different parts of the distribution function. The diamagnetic loop is sensitive to the perpendicular component $\mathrm{W}_{\text {dia }}=1.5 \mathrm{~W}_{\perp}$ while the equilibrium code EFIT [5] provides $\mathrm{W}_{\mathrm{MHD}}=0.75 \mathrm{~W}_{\perp}+1.5 \mathrm{~W}_{\|}$so combining the two gives total stored energy $\mathrm{W}=\mathrm{W}_{\perp}+\mathrm{W}_{\|}=$ $\left(2 * \mathrm{~W}_{\mathrm{MHD}}+\mathrm{W}_{\mathrm{dia}}\right) / 3$. Except in special circumstance such as ICH dominated heating, the anisotropy is 
small enough that it is better to use $\mathrm{W}=\mathrm{W}_{\mathrm{MHD}}$ due to offsets and noise affecting $\mathrm{W}_{\text {dia }}$ and this is what we utilise here.

\subsection{Smoothing the data}

The experimental data used in the power balance comes from systems which have very different time resolutions and different noise levels. Thanks to the JET central timing system jitter in synchronisation between diagnostics is not considered a problem. Most constraining are the measurements of plasma energy $\mathrm{W}_{\mathrm{MHD}}$ because the equilibrium calculation uses sensors inside and outside the vessel so that the skin time is involved. At worst, the time resolution / lag is about $\sim 5 \mathrm{~ms}$. Next in the list comes the bolometer system which has a time response of $\sim 1 \mathrm{~ms}$. Raw data from the principle diagnostics are shown in Fig. 1. The example pulse was chosen because it exhibits large changes in stored energy, radiated power (impurity event) and ELM frequency. The spikes in total radiated power associated with ELMs are very much smaller than were observed with the JET carbon wall. This is generally true and helps reduce the error in our dynamic power balance analysis. Despite questions over the reliability of the $\mathrm{W}_{\text {MHD }}$ derived from equilibrium analysis due to edge currents or anisotropy, the agreement with $\mathrm{W}_{\text {Dia }}$ is generally good as shown our example below but $\mathrm{W}_{\text {Dia }}$ tends to be noisier so $\mathrm{W}_{\mathrm{MHD}}$ is preferred. Power is calculated from the derivative of the plasma energy using the central difference $\left(\mathrm{W}_{\mathrm{i}+1}-\mathrm{W}_{\mathrm{i}-1}\right) / 2 \Delta \mathrm{t}$.

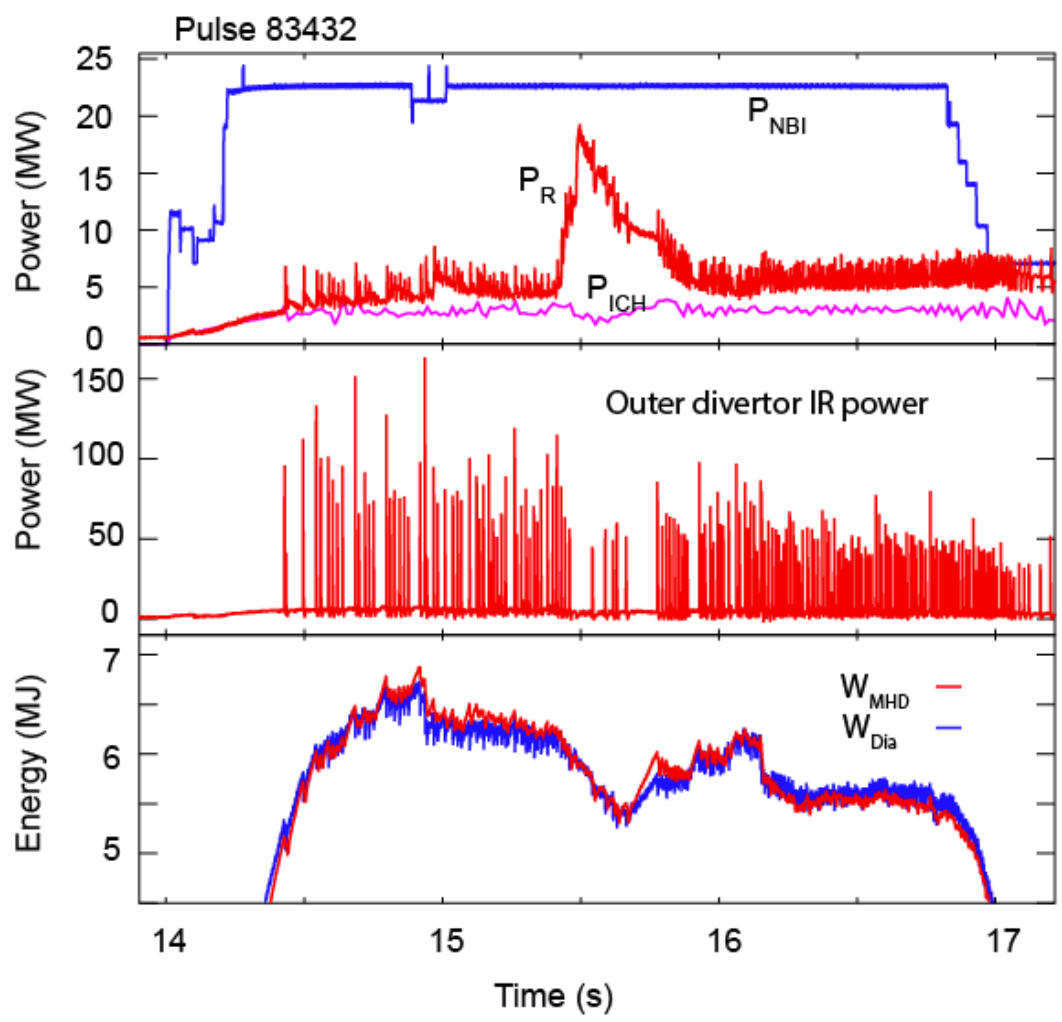

Fig. 1 Example of unsmoothed data used in the power balance analysis. Top frame: main power inputs and radiated power loss. Middle: outer divertor power derived from IR camera. Bottom: diamagnetic loop and MHD derived plasma energies.

To obtain a useful power balance we need to smooth the data from the different diagnostics to a common value. Initially, a simple box car smooth was used but this still leaves sharp steps when the box passes over discrete ELM events. A triangular smoothing kernel has therefore been used where the weight of contributing points decreases linearly with distance from the smoothing point. The quoted smoothing times correspond to the width of the base of this triangle. This smoothing algorithm conserves energy, which is essential, and is found to work reliably without noticeable phase lag for smoothing windows $\geq 25 \mathrm{~ms}$. A more sophisticated smoothing algorithm which better replicates the time response of the slowest measurement could potentially improve the useful time resolution. 


\subsection{Fitting the power balance}

The data sets we will focus on in this paper come from a series of JET experiments in which the scaling of divertor power width and ELM power deposition were studied in the ITER-like Wall and preceding carbon wall campaigns [6][7]. These discharges are good for our purposes because the plasma shape was very similar in all cases and the data span a wide range of input power magnetic field, $\mathrm{B}_{\mathrm{T}}$, and plasma current, $\mathrm{I}_{\mathrm{p}}$. The plasmas are attached so we do not expect the IR data to be polluted by broad band plasma emission. The outer strike point is resting on the central tile (Tile 5) in all cases. This tile was a made from a single block of carbon fibre composite (CFC) for the carbon wall phase but was changed to stacks of bulk tungsten lamellas for the ITER-like wall [8]. In both cases, the outer strike point is viewed from the top of the machine and high quality data are obtained [3][6][7]. We have selected pulses from these lists with $\mathrm{I}_{\mathrm{p}}$ in the range $1 \mathrm{MA}$ to $3.5 \mathrm{MA}$ and $\mathrm{B}_{\mathrm{T}}$ from $1 \mathrm{~T}$ to 2.8T. Time windows have been selected avoiding the main NBI switch on and switch off due to overshoots and undershoots in IR power that sometimes occur due to jumps in background light which are not yet understood.

The number of time points extracted from the smoothed data corresponds to two points per smoothing interval. In the ITER-like Wall data set, 35 pulses are fitted. Using the data smoothed to $100 \mathrm{~ms}$ this gives us 22,950 points. The procedure minimises the residuals between the measured IR power and that predicted by a rearrangement of equation (2). In Fig. 2, parameters $\mathbf{b}, \mathbf{d}$ and $\mathbf{e}$ have been fitted to give: $\mathbf{b}=0.96( \pm 0.006), \mathbf{d}=2.28( \pm 0.014)$ and $\mathbf{e}=1.00( \pm 0.006)$. Remaining parameters $\mathbf{a}, \mathbf{c}$ and $\mathbf{f}$ were fixed at unity. The error bars are impressively small but meaningless because it is clear from the clumping of the data points in the plot that we do not have a perfect model for the data because the deviations are not simply due to statistical noise. The simple representation of data by equation (2) only applies on average and there are other terms, systematic variations in the experiments or nonlinearities which would need to be characterised to improve the fit. Although in theory we should be able to fit all but one parameter e.g. a to $\mathbf{e}$ with $\mathbf{f}$ fixed, this produces large and physically unreasonable multipliers a $\left(\mathrm{P}_{\mathrm{Oh}}\right)$ and $\mathbf{c}\left(\mathrm{P}_{\mathrm{ICR}}\right)$. Once again this shows the limitations of equation (2).

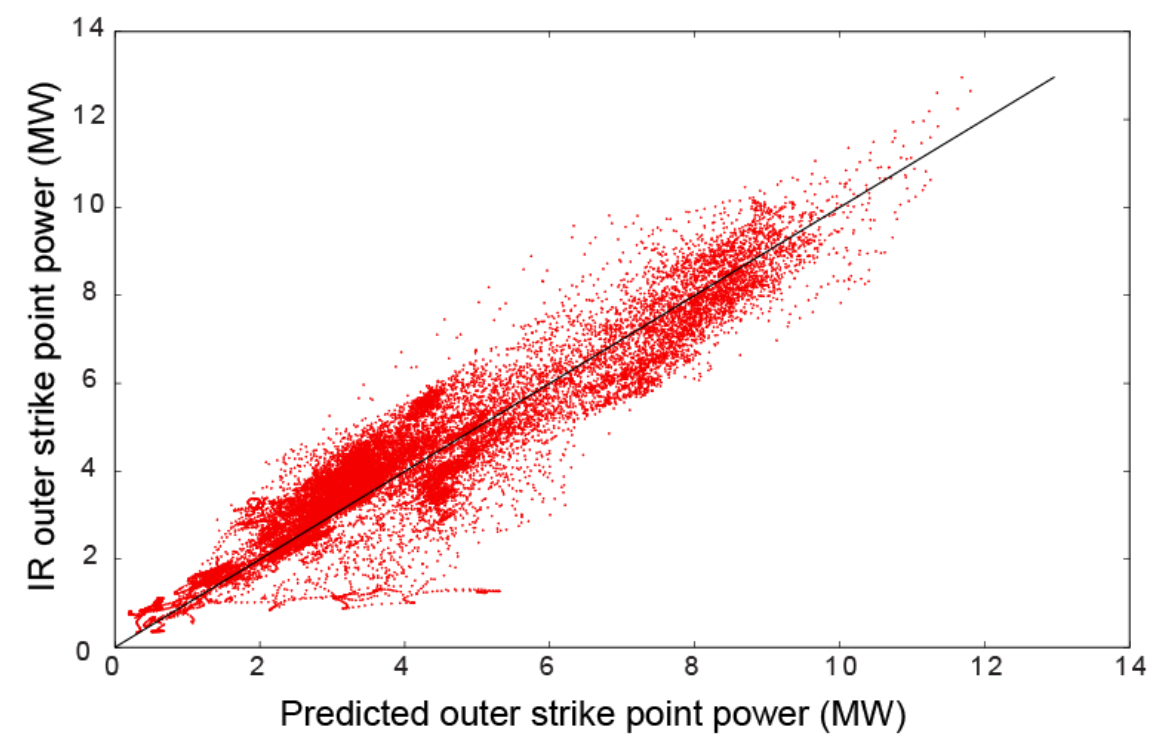

Fig.2 Power measured by IR camera at the outer strike point with $100 \mathrm{~ms}$ smoothing vs a least squares fit to parameters $b, d$ and e in equation (2): $P_{O h}+0.96 \times P_{N B I}+P_{I C H}=2.28 \times P_{I R}+1.00 \times P_{R}+d W / d t$

In Fig. 3 the absolute deviation between the outer strike point power and the fit has been binned and this shows that the number of extreme outliers is very small. However, the distributions also show skewness which again shows that the errors cannot be purely random noise. 


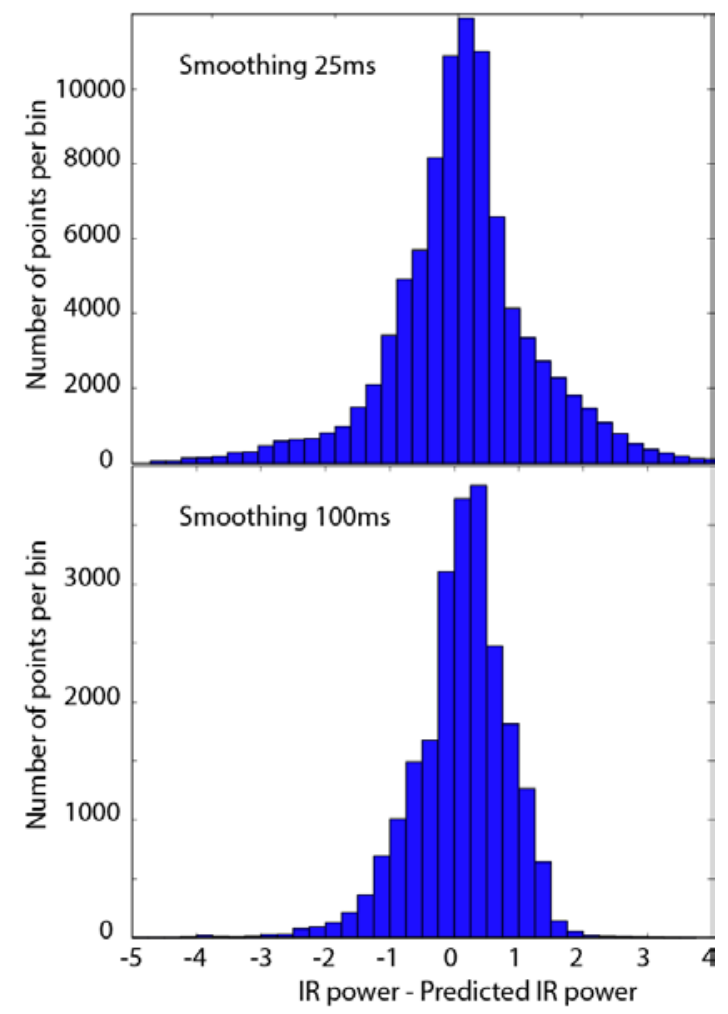

Fig. 3 Binned points showing the deviation from the best fit to the outer divertor IR power. Lower: same data and smoothing time as used in Fig. 2. Upper: smoothing time reduced to $25 \mathrm{~ms}$.

\subsection{Predicting the total plasma load on the PFCs}

While Fig. 1 shows significant scatter overall, there are many individual pulses in the set for which the fit is near optimal and excellent power balance over a wide time window is often observed. The best way we have found to represent this is to plot the total conducted plasma load which in this case is $\mathrm{P}_{\text {plasma }}=\mathbf{2 . 2 8} \times \mathrm{P}_{\mathrm{IR}}$ alongside its predicted value which for the fit of Fig. 1 is $\mathrm{P}_{\text {predicted }}=\mathrm{P}_{\mathrm{Oh}}+\mathbf{0 . 9 6} \times \mathrm{P}_{\mathrm{NBI}}+$ $\mathrm{P}_{\mathrm{ICH}}-\mathbf{1 . 0 0} \times \mathrm{P}_{\mathrm{R}}-\mathrm{dW} / \mathrm{dt}$. An example of such a plot is shown on Fig. 4 with a smoothing time of $25 \mathrm{~ms}$. Also shown on the plot are $-\mathrm{P}_{\mathrm{rad}}$ and $-\mathrm{dW} / \mathrm{dt}$ which are negative both for clarity and because they appear as losses in calculating $\mathrm{P}_{\text {predicted. }}$ The agreement is extremely good over the whole pulse despite large changes in $\mathrm{dW} / \mathrm{dt}$ and a radiation event due to high $\mathrm{Z}$ impurities. This shows that within a pulse at least, equation (2) can well describe the power balance over a wide dynamic range.

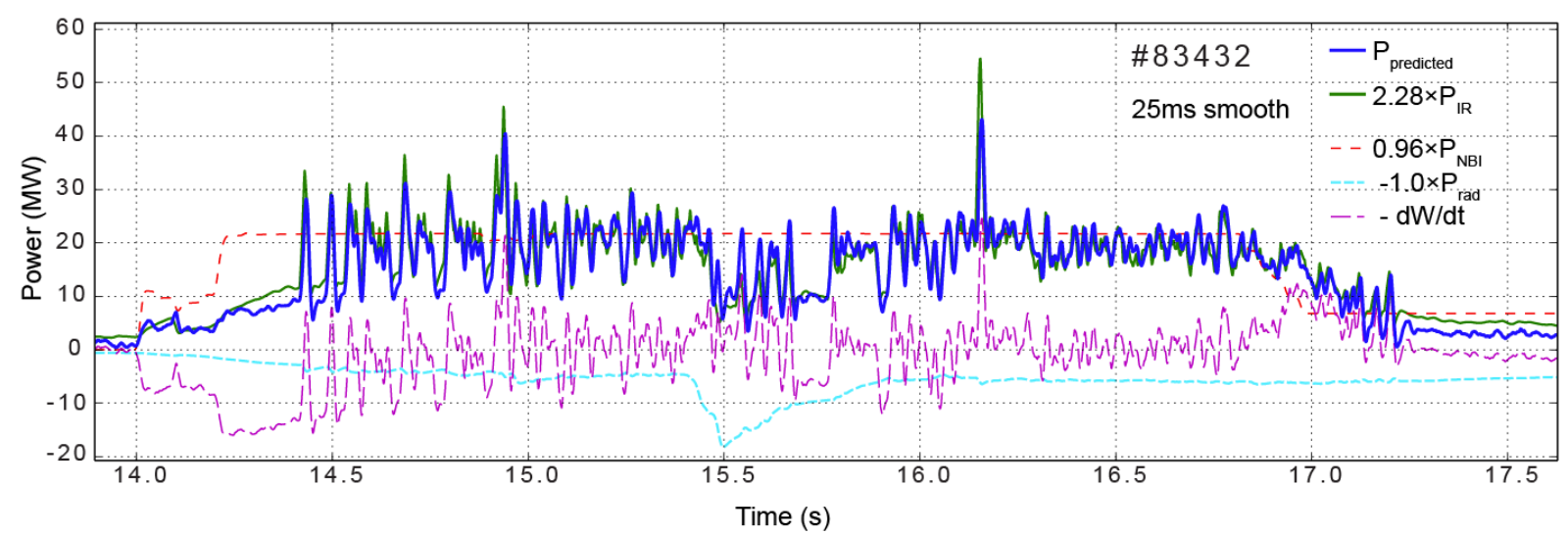

Fig. 4 JET pulse 83432 total predicted plasma load $P_{\text {predicted }}=P_{O h}+0.96 \times P_{N B I}+P_{I C H}-\mathbf{1 . 0 0} \times P_{R}-$ $d W / d t$ based on the fit of Fig. 1 and $P_{I R}$ plasma $=2.28 \times P_{I R}$ with which it should be compared. The triangular smoothing window is $25 \mathrm{~ms}$ for all signals. Not shown are $P_{I C H} \sim 3 M W$ and $P_{O h}<0.5 M W$ 
An important contribution from the changes in stored energy can be seen more clearly if we artificially switch off the dW/dt term as in Fig. 5. This plot also shows that the changes in average infra-red power during the high $\mathrm{Z}$ impurity event are very consistent with the radiated power. Also apparent are the large overshoot at the beginning of the pulse and undershoot at the end which are due to the rise and decay of the thermal stored energy at the beginning and end of the pulse. During the pulse, the spikes are due to ELMs but the power going into rebuilding the pedestal between ELMs can also be significant.

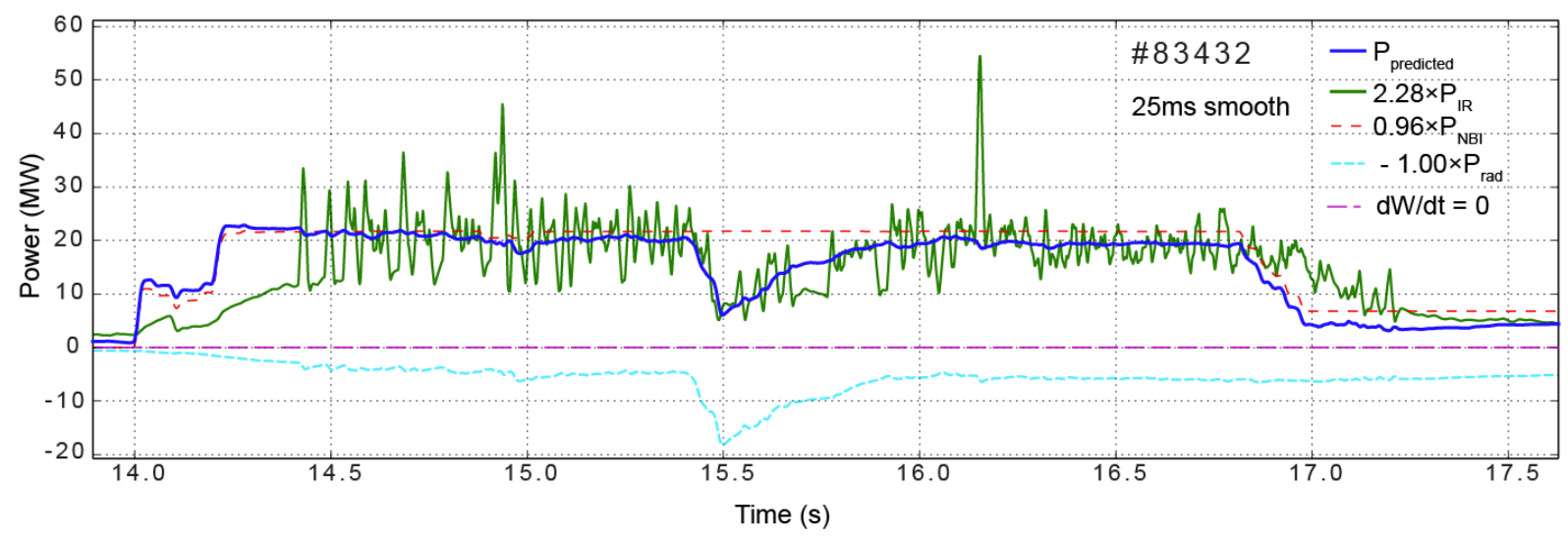

Fig. 5 Repeat of Fig. 4 but with the dW/dt term set to zero to illustrate the importance of changes in thermal stored energy for the power balance in JET. It is also clearer that the magnitude of the radiation event at $15.5 \mathrm{~s}$ is well captured i.e. the radiation multiplier is valid over a wide range.

Although not presented in this paper, similarly good agreement to that in Fig. 4 can be obtained using a new IR views of the outer and inner corners of the JET divertor. In each case the values of multiplier d which give good fits are consistent with the respective tile energy ratios from thermocouples $( \pm 10 \%)$.

We commonly use a smoothing time of $25 \mathrm{~ms}$ when looking at data such as that shown in Fig. 4 because we find empirically that this is usually sufficient to average out differences in diagnostic response functions. Of course, the appearance changes when the data is smoothed by different amounts. This is illustrated by Fig. 6 in which a one second segment of the pulse shown in Fig. 4 is expanded out and smoothed by $12 \mathrm{~ms}, 25 \mathrm{~ms}$ and $50 \mathrm{~ms}$. Significant deviations and phase lags start to become apparent with the $12 \mathrm{~ms}$ smooth.

In some of the pulses, two equivalent IR camera views were available in Octants 5 and 8 . Deviations between the two are $<10 \%$ which is consistent with the scatter seen in the toroidally distributed tile thermocouple data and within the expected uncertainty due to material property variations and calibration errors. The assumption of toroidal symmetry is therefore consistent with the data. 


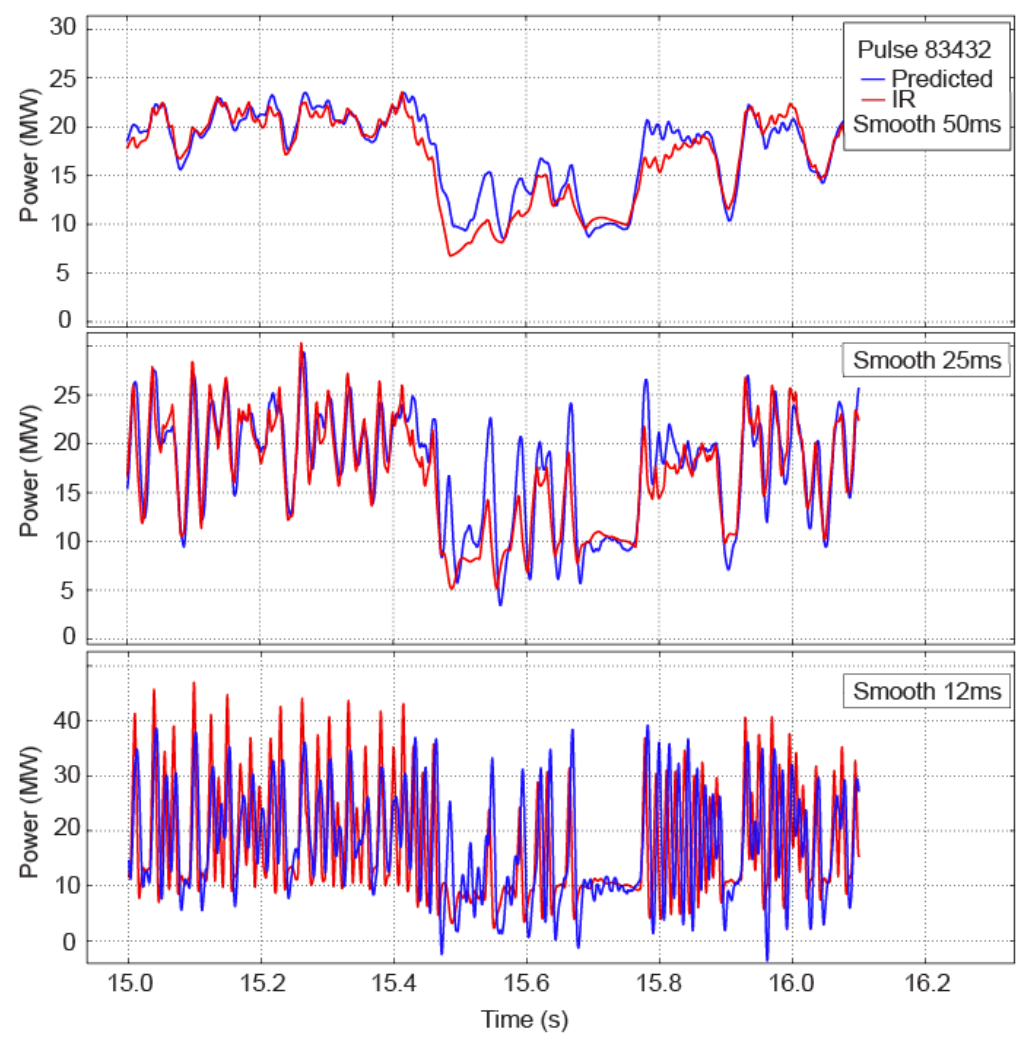

Fig. 6 Comparison of the effect of different smoothing times for the same pulse as Fig. 4. The IR power refers to the power measured on the outer divertor $\times 2.28$ which gives the expected total divertor power for comparison with $P_{\text {predicted }}=P_{O h}+0.96 \times P_{N B I}+P_{I C H}-1.00 \times P_{R}-d W / d t$ as before.

\subsection{A check against JET carbon wall data}

How do we know whether the multiplier which converts measured IR power to total divertor power is plausible for pulses run on Tile 5? The fit presented in section 2.2 for our ITER-like Wall data set gives $\mathbf{d}=2.28$ for this parameter. One way would be to use tile calorimetry and check the ratio of the energy arriving at the whole divertor to that on Tile 5 because the energies like the fitting process are averaging over many different conditions. Unfortunately for ITER-like Wall, Tile 5 is a complex assembly comprising 8000 bulk W lamellas [8] and the thermocouple data are not reliable. However, in the carbon wall phase of JET similar pulses were run as part of the scrape-off layer scaling studies for comparison to ITER-like Wall [6] and in this case Tile 5 was a large format CFC tile well suited to calorimetry. For these pulses, the average ratio of the total energy found on the whole divertor to that measured on Tile 5 is found to be 2.23 with a standard deviation of 0.27 . This sounds consistent but if the dynamic power balance analysis is applied to the carbon wall pulses (77,790 points, smoothing $100 \mathrm{~ms}$ ) then the equivalent fit to parameters $\mathbf{b}, \mathbf{d}$, e are: $\mathrm{P}_{\mathrm{Oh}}+\mathbf{0 . 8 8} \times \mathrm{P}_{\mathrm{NBI}}+\mathrm{P}_{\mathrm{ICH}}=\mathbf{1 . 9 9} \times \mathrm{P}_{\mathrm{IR}}+\mathbf{0 . 7 3} \times \mathrm{P}_{\mathrm{R}}$ $+\mathrm{dW} / \mathrm{dt}$. If we again fit the ITER-like Wall power balance but force parameters $\mathbf{d}=1.99$ to match the carbon wall power balance fit, then $\mathbf{b}=0.84$ and $\mathbf{e}=0.93$. Fixing also the radiation multiplier to its carbon wall value of $\mathbf{e}=0.73$ and again fitting the ITER-like Wall pulses gives us a neutral beam power multiplier of $\mathbf{b}=0.78$ (rather similar to some of the fits to the energy balance data [1]). The variation in $\chi^{2}$ between these cases is only $4 \%$ which illustrates the problem we have in identifying calibration issues or co-linear terms associated with individual elements of equation (2).

\section{Discussion and Outlook}

We have shown that a fit based on a linear model which assumes simple calibration factors or multipliers on each term can produce an impressively good power balance when applied to the time dependent data in individual pulses when triangular smoothing of $\geq 25 \mathrm{~ms}$ is applied. Provided the 
plasma shape is kept constant, the same parameters can be applied over a wide range of conditions in terms of radiated power, input power and thermal stored energy change. This is proving a very useful framework for checking of JET data consistency and spotting anomalies. It also shows the potential for real time calculation of total power loads. The smoothing applied means that individual ELMs cannot be studied on shorter timescales but it is still helpful in demonstrating the data consistency.

The agreement in the time series is better than expected given the uncertainties involved. Infra-red camera data comes from a single tile at the outer strike point and we are assuming that the power going elsewhere is simply proportional with a constant factor even during ELMs when smoothed over $25 \mathrm{~ms}$ with a triangular kernel. Plasma energy is also difficult to measure on short timescales due to issues such as edge currents, vessel screening and noise. Because the power is calculated from the derivative of the plasma energy the result is noise sensitive. These factors mean that plasma energy controls the minimum useful smoothing time. In this paper, we have tried to get away from the standard approach where discharges are broken down into quasi-steady state inter-ELM [11] and transient ELM phases [6]. Our aim is consider the consistency of the data at the maximum frequency allowed by the limitations of the diagnostics and associated algorithms. Our results show is that the approximation of steady conditions is seldom good, transients play a role in the power balance over a wide range of frequencies and stored energy changes are important between ELMs also.

The fitted factors required to convert total power on one tile to total conducted power, are reasonably consistent with energy ratios from thermocouple based tile calorimetry in the JET carbon wall campaigns where complete sets of measurement are available. However, while we can find combinations of parameters that provide good fits to particular power balance data sets, there are usually a number of equivalent but different combinations of parameter values giving similar $\chi^{2}$. We are therefore not able to unambiguously identify the cause of the energy balance deficit despite the additional constraint introduced by the stored energy changes in the power balance. Fits where power balance is achieved via the multiplier on IR power even make it hard to clearly demonstrate that there is a deficit in the power balance equivalent to that seen for energy.

The challenge for the future is that we need to reduce the uncertainties in the calibration or cross calibration of the main measurements to $<<10 \%$. Currently underway is an analysis of the rise in thermal stored energy after neutral beam switch-on (see Fig. 4 at 14s) which in conjunction with high time resolution analysis of shinethrough and other prompt losses, has the potential to check the consistency of the nominal neutral beam power against stored energy derived purely from magnetics measurements. In the future, experiments dedicated to simultaneous power and energy balance studies which optimise the diagnostic data and combine the necessary variations in the heating, radiated power and stored energy would also help to reduce the uncertainties.

\section{Acknowledgements}

This work has been carried out within the framework of the EUROfusion Consortium and has received funding from the Euratom research and training programme 2014-2018 under grant agreement No 633053. The views and opinions expressed may not reflect those of the European Commission. This work was also part-funded by the RCUK Energy Programme under grant EP/P012450/1. To obtain further information on the data and models underlying this paper please contact PublicationsManager@ccfe.ac.uk.

\section{References}

[1] G.F.Matthews et al., Nuclear Materials and Energy (2016) http://doi.org/10.1016/j.nme.2016.12.012

[2] G.F.Matthews et al., 2011 Phys. Scr. 2011014001

[3] I.Balboa et al., Review of Scientific Instruments, 10D530 (2012) 83

[4] L.C. Ingesson, JET report JET-R(99)06

[5] L.L.Lao et al., Fusion Science and Technology / Vol. 48 No. 2 (2005) 968-977

[6] T.Eich et al., Journal of Nuclear Materials 438 (2013) S72-S77

[7] B.Sieglin et al., Plasma Phys. Control. Fusion 55 (2013) 124039 (8pp) 
[8] Ph.Mertens, Phys. Scr. T145 (2011) 014002 (7pp)

[9] J.C.Fuchs et al., Journal of Nuclear Materials 337-339 (2005) 756-760

[10] J.-C.Vallet et al., 30th EPS Conference on Contr. Fusion and Plasma Phys., St. Petersburg, 711 July 2003 ECA Vol. 27A, P-1.137

[11] A.W.Leonard et al., Journal of Nuclear Materials 220(1995)325

[12] R.P. Wenninger Nucl. Fusion 54 (2014) 114003 (8pp)

[13] M.Wischmaier, J. Nucl. Mater. 463 (2015) 22-29 